\title{
Optimization of car frontal protection system
}

\author{
M. Pohlak ${ }^{\mathrm{a}}$, J. Majak and M. Eerme \\ Dept. of Machinery, Tallinn University of Technology Ehitajate tee 5, 19086 Tallinn, Estonia
}

Received 20 August 2007; accepted 15 October 2007

\begin{abstract}
Aim of the current study is to design the extra frontal protection system of a car satisfying the requirements of directive 2005/66/EC of the European Parliament and of the Council. The car frontal protection system is treated as additional energy absorbing element. An analysis of a car-pedestrian crash situation is performed by use of explicit Finite Element Analysis solver and the following stiffness analysis with implicit Finite Element Analysis solver. For modeling response surface and search for optimal solution the LS-OPT software is utilized and some comparative simulations are performed by use of MATLAB. The experimental validation of the models designed is performed.
\end{abstract}

Key words: Crash safety; impact energy absorption; multicriteria optimization

\section{Introduction}

Car frontal protection systems can be categorized as original equipment or as separate technical units. Both systems provide additional frontal protection for motor vehicles, but constitute potential risk to the safety of pedestrians. More than 8.500 pedestrians and cyclists die and over 290.000 are injured in road crashes in the EU per year (Dörr et al. [1]). This forms about $1 / 5$ of total victims in traffic accidents. For that reason, safety remains a top priority for the auto industry today. In order to develop safer cars the crash engineers have begun to redesign the bumper, hood and etc. with the aim to make them better energy absorbers (Du Bois et al. [2], Lawrence et al. [3], Matsui [4], Schuster [5]). In Du Bois et al. [2] a comprehensive overview on vehicle design safety is given. The basic concepts of vehicle safety, design and the evolution of body structures, a theoretical background and finite element modeling methods of the vehicle body design technology, analytical methods of determining occupant motion and some biomechanics issues of human injury are covered. In Lawrence et al. [3] the benefits of the brake assist system are analyzed. The effects of vehicle bumper height and impact velocity on the type of lower extremity injury are studied by Matsui [4]. It is pointed out that the main injury at an impact velocity of around $20-30 \mathrm{~km} / \mathrm{h}$ is to the knee ligament, but at an impact velocity of around $40 \mathrm{~km} / \mathrm{h}$, a fracture of the lower extremities. The paper by Schuster [5] is devoted to the bumper system design for pedestrian impact. Different approaches for reducing the severity of pedestrian lower limb impacts are discussed. More popular cushioning methods are reviewed. Some trends in bumper design, as the use of lower stiffeners, alternative energy absorbers,

${ }^{a}$ Corresponding author: meelisp@staff.ttu.ee beam face features, flexible beams and add-on structures are pointed out.

A number of papers are devoted to impact energy absorbers (Al Galib et al. [6], Alghamdi [7], Gupta [8], Griškevièius et al. [9], de Kanter [10], etc.). In Al Galib et al. [6] the crash behavior of circular aluminum tubes undergoing axial compressive loading is studied experimentally and numerically. Static and dynamic behavior of circular thin-walled tubes is considered, the values of the mass and impact velocity of tubes are varied. Axisymmetric and mixed deformation modes are analyzed. Numerical results obtained from FE analysis are validated against static and dynamic test results. In Alghamdi [7] common shapes of collapsible energy absorbers including circular tubes, square tubes, frusta, struts, honeycombs, and sandwich plates are reviewed. Axial crushing, lateral indentation, lateral flattening, inversion and splitting are considered as possible deformation modes. The viability of using structural foam in B-pillar and bumper designs is investigated by Gupta [8]. It is concluded that the B-pillar and the rear bumper are potential areas where structural foam could replace steel and other materials and allow weight reduction in comparison with respective base models. In Griškevièius et al. [9] the impact energy absorption of the vehicles front structures during frontal crash is studied. The energy absorbing capabilities of longeron columns under axial compression loading are analyzed. It is pointed out that the degradation of the structures in the old cars has significant influence on the energy absorbed. Capability to absorb up to three times more energy by modern AVC longeron columns than corroded longeron columns in the old cars is reported. Testing and numerical simulation of multimaterial energy absorbers is performed by de Kanter [10]. The properties of the metallic and fibre reinforced plastic 
(FRP) cylinders in the crushing behavior are discussed. It is stated that multi-material elements demonstrate both, metallic and composite characteristics in crushing behavior i.e. metallic and composite materials are complementing each other. Three different possibilities for integrating metal and composite materials are analyzed (reinforcement the metal cylinder with FRP, reinforcement the FRP cylinder with metal, creating a multi-material component as one). The optimal combination of materials, the fibre orientations, number of fibre layers and the thickness ratios are considered as design parameters in a multi-material element design process. In the case of deformation of the multi-material element up to failure the metal progressive plastic collapse and the progressive brittle fibre reinforced plastics (FRP) collapse mechanisms apply in combined form.

Employment of various optimization techniques is one of the trends in car frontal protection system design (Hörmann [11], Wang [12]). In Hörmann [11], a cylinder impact on rigid wall is considered as an example. This paper is focused on the description of the capabilities of LS-OPT software.

Successive response surface methodology (SRSM) based optimization and its application to structural design is discussed in detail. Special attention is paid to crashworthiness analysis. Shape optimization of a crashbox using HyperMorph and LS-OPT software is studied by Wang [12]. The smoothness of the force values during the folding process of the crashbox is considered as a quality criterion. The difference between maximum and minimum force values of the force-intrusion is minimized.

In the current paper, the possibilities to increase safety of the car frontal protection system in accidents with pedestrians are studied. Main attention is paid to the design of fastening components. The stiffness of the components is limited by pedestrian safety and required structural stiffness of the car accessories. On one hand, the stiffness of the accessories mounted on the car front protection system (e.g. extra lights) must be high enough to withstand the vibrations and accelerations of the car. On the other hand, the stiffness must not be too high in order to avoid excessive accelerations of human body parts in case of accident. In order to obtain maximum energy absorption that is smooth enough, search for optimal configuration of support components of the structure is performed.

In the current paper, the emphasis is on the study of the frontal protection system of vehicle consisting of tubular accessory and the fastening components. The system is simply added by bolts to a standard motor vehicle without major modifications to the vehicle. It is important that the original energy absorbing structures of the vehicle will remain in place without alteration.

\section{Impact test}

In general, the tubular extra accessories, mounted to the front of vehicle, constitute a risk to the safety of pedestrians in the event of a collision. At the same time these

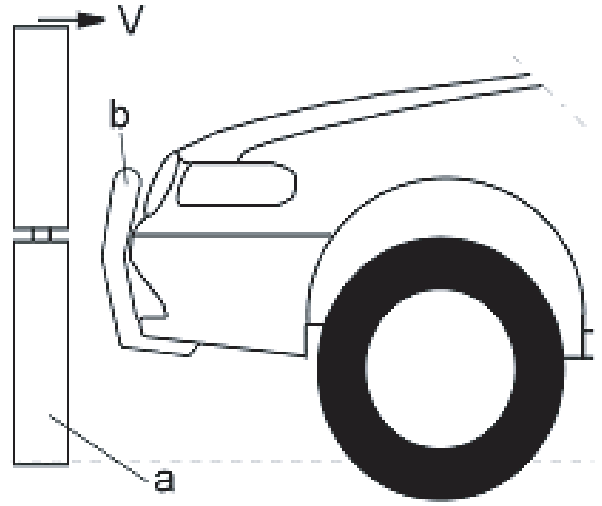

Fig. 1. Lower legform impact testing. (a) Legform impactor, (b) Frontal protection system, V - velocity of impactor.

systems have been still used in the EU in recent years. In order to protect pedestrians against such a risks, corresponding test methods and tools were developed by EEVC (European Enhanced Vehicle safety Committee) and elaborated by ACEA (Association des Constructeurs Europeens d' Automobiles) (Dörr et al. [1]).

According to the directive 2005/66/EC (Directive [13]) the car frontal protection systems must pass the following tests:

- lower legform test;

- upper legform test (required for systems with height over $500 \mathrm{~mm}$ );

- upper legform leading edge test (to bonnet, for monitoring purposes only);

- child/small adult headform test (to bonnet).

In the current study, it is assumed that the height of the car frontal protection system designed is less than $500 \mathrm{~mm}$ and main attention is paid to the safety requirements proceeding from lower legform test. The test is depicted in Figure 1. In the test, the impactor ((a) in Fig. 1) is shot at the speed of $11.1 \mathrm{~m} / \mathrm{s}$ at the frontal protection system of the vehicle. There are three types of sensors mounted inside the impactor: acceleration sensor, bending angle sensor and shear displacement sensor. For all three, there are certain limits defined by the directive 2005/66/EC (Directive [13]) as:

- the maximum dynamic knee bending angle shall not exceed $21.0^{\circ}$;

- the maximum dynamic knee shearing displacement shall not exceed $6.0 \mathrm{~mm}$;

- the acceleration measured at the upper end of the tibia shall not exceed $200 \mathrm{~g}\left(g=9.81 \frac{\mathrm{m}}{\mathrm{s}^{2}}\right)$.

It is assumed above that the total permissible mass of the vehicle is less than $2500 \mathrm{~kg}$. In the case where the total permissible mass of the vehicle exceeds $2500 \mathrm{~kg}$, the corresponding maximum values of the knee bending angle, knee shearing displacement and acceleration measured at the upper end of the tibia are $26.0^{\circ}, 7.5 \mathrm{~mm}$ and $250 \mathrm{~g}$, respectively. 


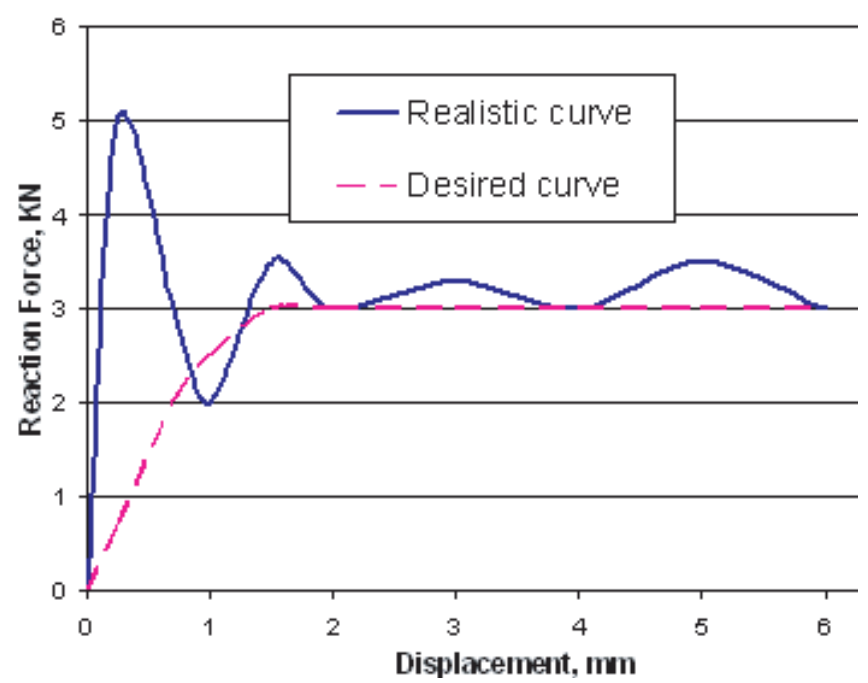

Fig. 2. Force-displacement diagram of energy absorbing component: realistic curve and desired curve.

While with bending angle and shear displacement it is easier to fit within the given limits, with the acceleration limit, the situation is more complicated.

\section{Impact energy absorption}

From the literature, different kind of energy absorbing structures (rings, thin walled members, laminates, honeycombs and etc.) can be found, materials vary from solid metals to composites and cellular materials (Alghamdi [7], de Kanter [10], Lu et al. [14]). Unfortunately, most structures absorb energy in an unstable manner. When impact loading starts, there will be a high peak of reaction force followed by smaller peaks or more constant level of reaction forces (Fig. 2). A more desirable situation would be, if the reaction force increased steadily to some predefined level and remains constant at this level ( $\mathrm{Lu}$ et al. [14]). In the deceleration of the impactor, only few components are involved. They are tubular parts, fastening components and at the final stage, the bumper of the car. To lessen the acceleration, optimization of tubular parts and fastening components have to be addressed. In Figure 3 the acceleration diagram obtained from the lower legform impact test is shown (corresponds to initial design of the fastening component). As it can be seen, the acceleration is too high, so the design does not conform to the requirements of directive 2005/66/EC (frontal protection system is too stiff).

In the current study, the detailed optimization of the tubular parts is omitted. The properties of the tubes are selected as appropriate as technologically possible (light structure, thin walls, etc). Main attention is paid to optimization of the geometrical parameters of fastening components. The initial configuration of the fastening components is provided by the manufacturer of the car frontal protection system. The current study covers design improvement rather than topology optimization, since the

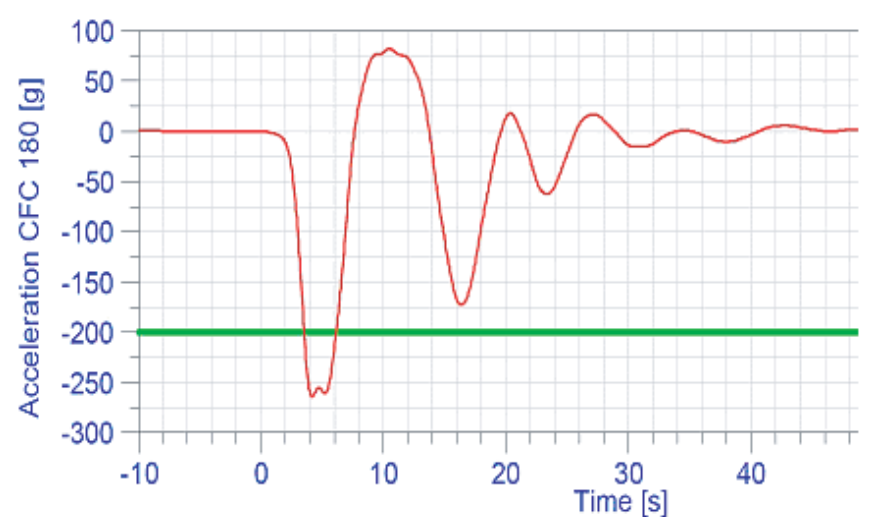

Fig. 3. Acceleration plot of legform impact test.

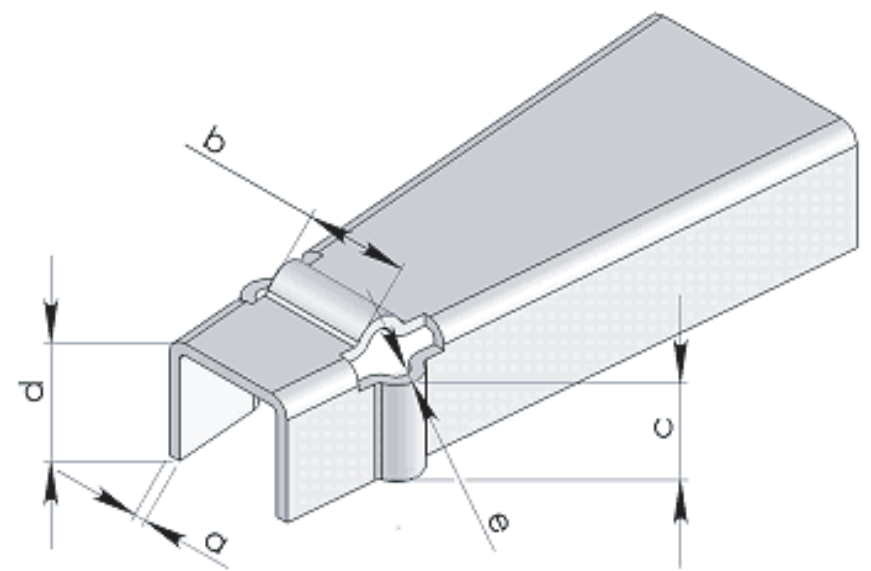

Fig. 4. Energy absorbing component.

values of the design parameters corresponding to predefined initial topology of the component are determined (some modifications concerning topology of the component are available). A simple energy absorbing component is given in Figure 4. The geometrical parameters $a$, $b, c, d$ and $e$ shown in Figure 4 are considered as design variables.

\section{Formulation of optimization problem}

The maximization of the internal energy and minimization of the mass are most commonly used as objectives for crashworthiness optimization problems. Definitely, the criteria of interest in crashworthiness studies are not limited by the two formulations mentioned above. In the following, two different optimality criteria are discussed. In order to cover different criteria and constraints, the optimization problem is formulated as a general nonlinear optimization task as

$$
\min f(\vec{x})
$$

subjected to

$$
\begin{aligned}
g_{j}(\vec{x}) \leqslant 0, & j=1, \ldots m \\
h_{k}(\vec{x})=0, & k=1, \ldots l
\end{aligned}
$$


In (1)-(3) $f, g$ and $h$ are functions of independent design variables $x_{1}, x_{2}, \ldots x_{n}$ and

$$
\vec{x}=\left(x_{1}, x_{2}, \ldots x_{n}\right) .
$$

The objective functions considered in the current paper can be expressed as

a) minimization of the peak force (peak acceleration)

$$
f_{1}(\vec{x})=\max \left(F_{Z}\right),
$$

b) minimization of the difference between maximum and minimum force values

$$
f_{2}(\vec{x})=\max \left(F_{Z}\right)-\min \left(F_{Z}\right),
$$

where $F_{Z}$ stands for axial force component (in moving direction).

Theoretically, the optimality criterion (6) is correct, but its practical application for the posed problem is complicated. Namely, at initial stage $F_{Z}=0$ and the optimality criterion (6) reduces to criterion (5), if it is applied directly (see Fig. 2). In order to overcome the latter complication, the following two approaches are considered:

- criterion b) can be applied for time $t$ such that $t>t_{l}$, where the time value $t_{l}$ corresponds to the first peak of the force component $F_{Z}$;

- the term $\min \left(F_{Z}\right)$ in criterion (6) can be replaced by final value of the force component $F_{Z}\left(F_{z f i n a l}\right)$.

Obviously, the first approach can be applied without losing generality, but the second approach can be applied for certain kinds of problems only. Note that in the case of the above posed problem both approaches considered lead to the same results.

The simultaneous minimization of objective functions (5) and (6) leads to the multicriteria optimization problem. The corresponding functional, subjected to minimization, can be written as

$$
f(\vec{x})=\left[\max \left(F_{Z}\right), \max \left(F_{Z}\right)-\min \left(F_{Z}\right)\right] .
$$

Most commonly the multicriteria optimization problem (MOP) is solved by combining the multiple objectives into one scalar objective whose solution is a Pareto optimal point for the original MOP i.e. functional (7) can be replaced by the following functional

$$
f(\vec{x})=w_{1} f_{1}(\vec{x})+w_{2} f_{2}(\vec{x}) .
$$

In (8) $w_{1}$ and $w_{2}$ stand for the weights of objective functions $f_{1}$ and $f_{2}$ defined by relations (5) and (6), respectively.

A number of alternative techniques are available for solving multicriteria optimization problem, such as goal programming, homotopy techniques, normal-boundary intersection, multilevel-programming and etc. (Das [15]).

The constraints considered in the current model can be outlined as:

a) Linear constraints on design variables.
The set of design variables and the constraints laid on these variables depend on the structure and topology of the car frontal protection system considered. In general form, the constraints can be written as

$$
x_{i}<=x_{i}^{*}, \quad-x_{i}<=-x_{i}^{* *},
$$

where $x_{i}^{*}$ and $x_{i}^{* *}$ stand for upper and lower bounds of the design variable $x_{i}$, respectively.

b) Nonlinear constraint on displacements in $x-y$ plane (car is moving in $z$-axis direction).

As mentioned above, the stiffness of the components of the car frontal protection system is limited by pedestrian safety and required structural stiffness of the car accessories. The protection system designed must work as a good energy absorber, but at the same time it must be stiff enough with respect to loading in directions perpendicular to car moving direction. The weight of the system itself can be considered as acting load. The constraint providing the required stiffness of the car frontal protection system can be expressed in terms of displacements as

$$
u_{c}=\sqrt{u_{2}^{2}+u_{3}^{2}}<=u^{*},
$$

where $u_{2}$ and $u_{3}$ stand for the displacements in $x$ and $y$ direction (i.e. perpendicular to moving direction of the car), respectively and $u^{*}$ is a given limit value. It should be noted that the constraint (10) must hold good in normal car exploitation conditions and may be violated in the case of accident.

\section{Necessary optimality conditions}

First note that the equality constraints (3) can be written in terms of inequality constraints as

$$
h_{k}(\vec{x})<=0, \quad-h_{k}(\vec{x})<=0, \quad k=1, \ldots l .
$$

Let us introduce the extended functional $J_{*}$ as

$$
J_{*}=f(\vec{x})+\lambda^{T} g(\vec{x}) .
$$

In (12) $\lambda$ stand for Lagrange multiplier vector and the equality constraints (3) are included in inequality constraint vector $g(\vec{x})$.

Equalizing the total variation of the functional $J_{*}$ to zero, one obtains the necessary condition for the stationary value of the functional $J_{*}$, which are known as Karush-Kuhn-Tucker conditions:

$$
\begin{gathered}
\frac{\partial f(\vec{x})}{\partial \vec{x}}+\lambda^{T} \frac{\partial g(\vec{x})}{\partial \vec{x}}=0, \\
\lambda^{T} g(\vec{x})=0, \\
g(x)<=0, \\
\lambda>=0 .
\end{gathered}
$$

Gradient based optimization methods require computation of the first order derivatives of objective function $f(\vec{x})$ and constraints function $g(\vec{x})$ with respect to the design variables $\vec{x}$. Detailed solution procedure for the posed optimization problem is given in the next section. 


\section{Solution procedure}

In the case of the optimization problem considered, an application of the optimality conditions (13) is cumbersome, since the objective function (8) and nonlinear constraint function (10) are evaluated from dynamic and static FE analysis, respectively. The gradients can be calculated using an adaptive finite-difference method.

The solution procedure proposed can be decomposed into following subtasks:

a) design of simulation data, the set of the design variables must be prepared for a number of crash simulations,

b) dynamic FE analysis (explicit FEM) - crash simulation,

c) static FE analysis (implicit FEM) - stiffness analysis,

d) application of the response surface method (RSM),

e) search for optimal solution.

The simulation data are obtained by varying the design variables in limits given by (9).

Uniform grid can be used as initial approach if no other considerations are available. The content of the subtasks b)-e) is described in following sections.

\subsection{Finite element analysis (FEA)}

Numerical analysis is performed in LS-DYNA program by use of fully integrated shell elements (LS-DYNA [16]). The stress-strain behavior is modeled with multi-linear approximation. In order to consider plastic anisotropy the Hill's second order yield criteria is employed.

As mentioned above, the FEA is performed separately for crash simulation and stiffness analysis. The total number of simulations depends on number of design variables and on grid density, fixed at the stage of simulation data design. The number of design variables used in the case of different approaches was from 4 up to 8 . The dynamic and static analysis is performed with the same sets of the simulation data in order to get a complete set of output data. The output data used if further optimization procedure contains maximum values of the frontal force component (obtained from the dynamic analysis) and displacements in $x-y$ plane $u_{c}$ (evaluated by use of formula (10), where $u_{1}$ and $u_{2}$ are obtained from the static analysis). Obviously, in the case of objective function (8) the values of both expressions $\max _{t>0}\left(F_{Z}\right)$ and $\max _{t>t_{0}}\left(F_{Z}\right)-\min _{t>t_{0}}\left(F_{Z}\right)$ should be included in output data prepared for optimization procedure.

\subsection{Response surface method (RSM)}

Let us proceed from the predetermined set of designs introduced for FE analysis. In the following, the output data obtained from $\mathrm{FE}$ analysis are treated as response values. In RSM, the design surface is fitted to the response values using regression analysis. Most commonly, least squares approximations are used for this purpose. In the current paper, the generalized regression neural networks (NN) is used for the surface fitting. The surface constructed by use of NN do not normally contain the given response values (similarity with least-squares method in this respect). An approach proposed is based on the use of the MATLAB neural network toolbox and LS-OPT software (Stander [17]). The function newgrnn creates a two-layer network (MATLAB [18]). The first layer has radbas neurons and the second layer has purelin neurons. The response surfaces are generated simultaneously (with one call to newgrnn) for all response quantities $\left(x-y\right.$ displacement $u_{c}$ and objective function(s)). In order to calculate outputs for a concurrent set of values of the design variables, a network simulation function sim is used in MATLAB (NN Toolbox).

Similar two layer (one hidden layer) network is generated also in FE software LS-OPT for composing response surface. The LS-OPT software based approach is preferred in the current study, since it is compatible with LS-DYNA.

\subsection{Search for optimal solution}

Let us proceed from the surface modeled by use of neural networks. In order to determine the minimum of the objective function (8), the LS-OPT software has been utilized. The response surfaces for function $f(\vec{x})$ determined by formula (8) and $x-y$ displacement $u_{c}$ are treated as objective and constraints functions, respectively. Alternatively, some simulations have been performed by use of MATLAB function fmincon (Optimization Toolbox), but, as already mentioned above, the LS-OPT software is preferred due to its compatibility with LS-DYNA. The obtained results are found to be close, but in the case of both approaches, the convergence to the nearest extreme value of the objective function can be followed. Thus, the global extremes of the objective function can be achieved by the selection of a suitable initial point. In the case of the optimization problem considered, the initial point is chosen from the set of grid points as a point with minimal value of the objective function (search for minimum).

Obviously, the search for global optimum can be refined (or simplified) by applying genetic algorithm (at least at first stage of the search). However, in the latter case, the benefits of the integrated use of LS-DYNA and LS-OPT software cannot be exploited.

\section{Numerical and experimental results}

In Figure 5 the frontal force component $F_{z}(t)$, corresponding to initial and optimal sets of design variables, is given, respectively. Obviously, the maximal value of the $F_{z}(t)$ is reduced significantly (more than 4 times). All constraints are fulfilled in the case of both designs. Note that energy absorption is twice higher in the case of initial design. This can be explained with reduced dimensions of the component. It can be seen from Figure 5 that the shape of the 


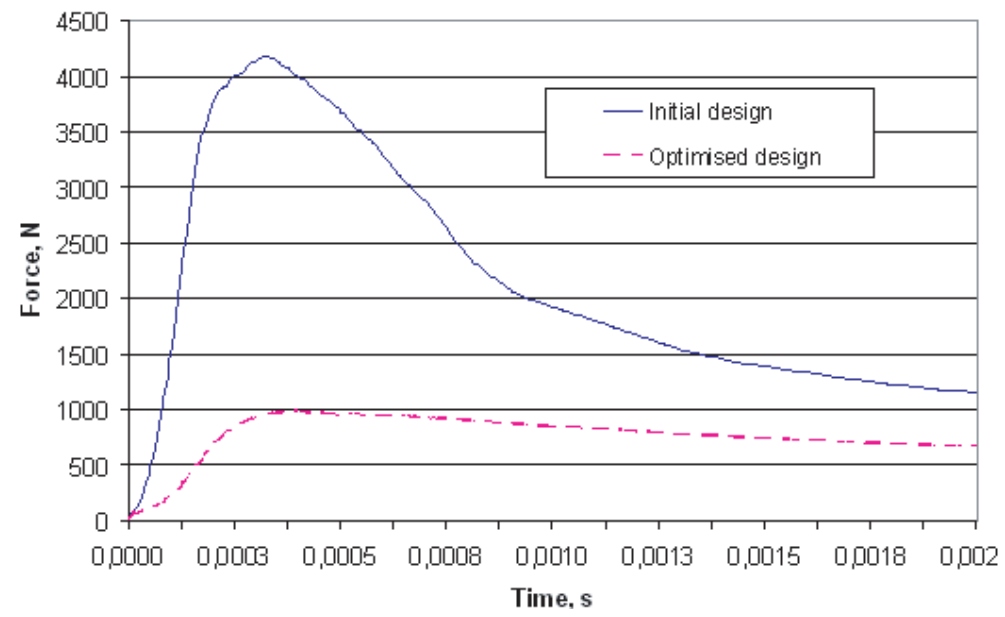

Fig. 5. Force - Time diagram: initial design and the optimized design.
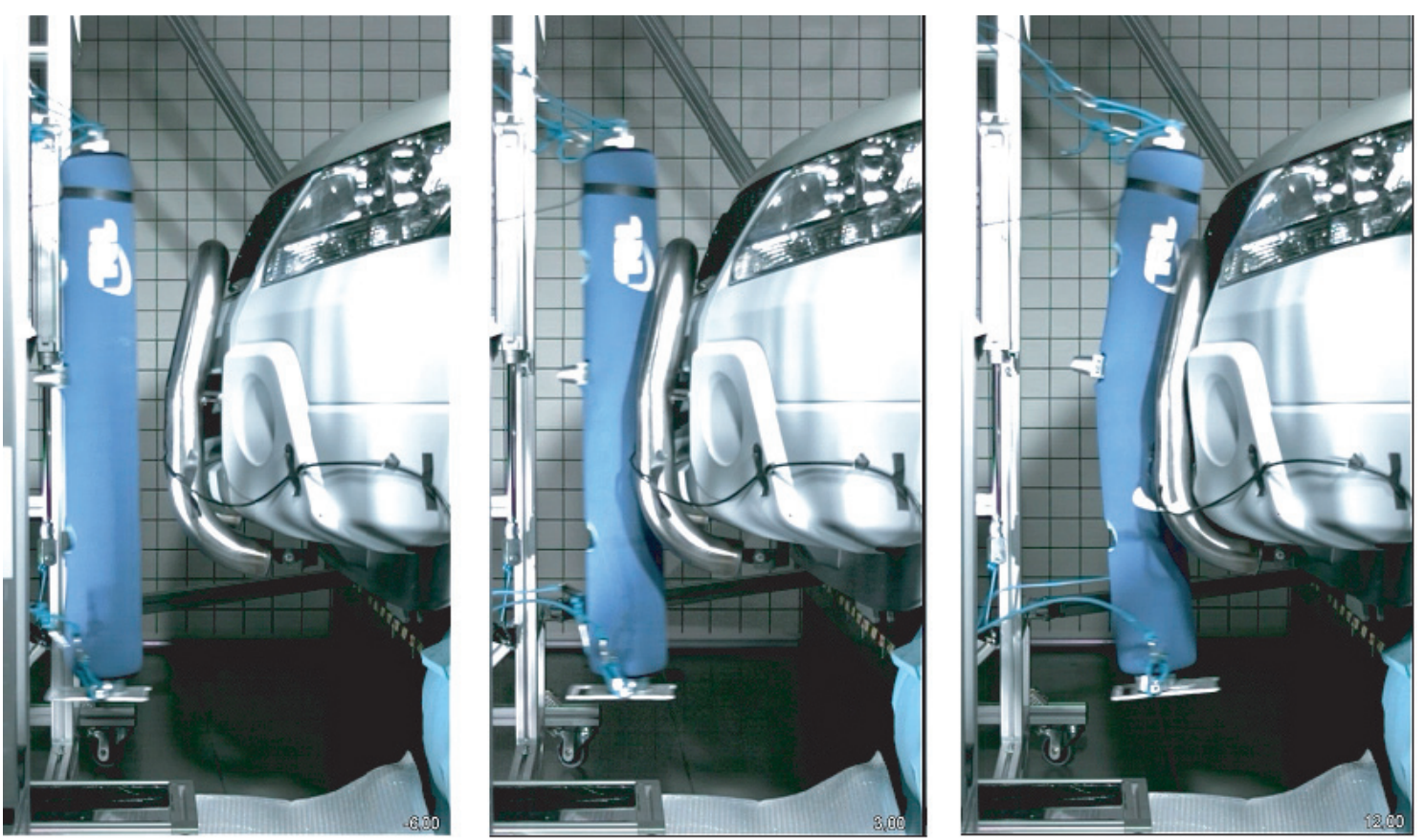

Fig. 6. Lower legform impact test.

force curve corresponding to the optimal design is quite similar to the shape of the desired force curve, depicted in Figure 2.

In order to validate the FEA models, an experimental study was carried out. Several configurations of the component shown in Figure 4 were tested. In Figure 6, the lower legform impact tests according to the directive 2005/66/EC (Directive [13]) to benchmark the initial design are shown. Figure 6 consist from three pictures chosen for describing lower legform impact test. First picture is done 6.3 milliseconds before contact, second and third pictures 3 and 12 milliseconds after contact, respectively. The forces and deformation modes determined from experiments were found to be reasonably close to that calculated by FEA.

\section{Conclusion}

Design optimization of a car frontal protection system is performed. Main attention is paid to the optimal design of the fastening components. A multicriteria optimal design problem is formulated and the necessary optimality conditions are derived. The design procedure proposed contains an analysis with dynamic loading for car-pedestrian crash situation by use of LS-DYNA explicit solver, following stiffness analysis (LS-DYNA implicit solver), response surface modeling and search for optimal design (LS-OPT, some comparative simulations are performed in MATLAB). The obtained numerical results are found to be in agreement with experimental results.

Unfortunately, the solution is sensitive with respect to initial design. In order to avoid problems with selection 
of the initial design (convergence to local minimum), the genetic algorithm (GA) based optimization technique can be applied. In the latter case, the FEA results should be transferred into alternative software. In future MATLAB and its toolboxes (optimization, genetic algorithm and direct search) are planned to be utilized for this purpose.

Acknowledgements. The work has been supported by Estonian Science Foundation grant G6835.

\section{References}

1. S.D. Dörr, H. Chladek, A. Hub, Crash Simulation in Pedestrian Protection. In: $4^{\text {th }}$ European LS- DYNA Users Conference. Occupant II/ Pedestrian Safety. Ulm, Germany, May 2003. p. 19-28

2. P. Du Bois, C.C. Chou, B.B. Fileta, T.B. Khalil, A.I. King, H.F. Mahmood, H.J. Mertz, J. Wismans, Vehicle Crashworthiness and Occupant Protection, American Iron and Steel Institute, Southfield (2004), p 368

3. G.J.L. Lawrence, B.J. Hardy, J.A. Carroll, W.M.S. Donaldson, C. Visvikis, D.A. Peel, A study on the feasibility of measures relating to the protection of pedestrians and other vulnerable road users - Final report, In: Project report, EC Contract No. FIF.20030937 (2004)

4. Y. Matsui, Effects of vehicle bumper height and impact velocity on type of lower extremity injury in vehiclepedestrian accidents. Acc. Anal. Prev. 37, 633-640 (2005)

5. P.J. Schuster, Current Trends in Bumper Design for Pedestrian Impact, In: SAE Technical Papers No. 200601-0464 (2006)

6. D. Al Galib, A. Limam, Experimental and numerical investigation of static and dynamic axial crushing of circular aluminum tubes. Thin-Walled Structures 42, 1103-1137 (2004)
7. A.A.A. Alghamdi, Collapsible impact energy absorbers: an overview. Thin-Walled Structures 39, 189-213 (2001)

8. S. Gupta, Using CAE to evaluate structural foam alternatives in B-pillar and bumper designs. In: $3^{\text {rd }}$ European LS-DYNA users conference, Paris, France (2001)

9. P. Griškevièius, A. Žiliukas, The impact energy absorption of the vehicles front structures. Transport 18, 97-101 (2003)

10. J. De Kanter, Energy absorption of monolithic and fibre reinforced aluminium cylinders, Delft University of Technology, PhD Thesis (2006), p. 219

11. M. Hörmann, A. Schulz, W. Rust, Structural Optimization using LS-OPT: Basics and Examples. In: Proceedings of 21st CAD-FEM Users' Meeting, Potsdam (2003)

12. H. Wang, H. Müllerschön, T. Mehrens, Shape Optimization of a Crashbox using HyperMorph and $L S-O P T$. In: Proceedings of 4th German LS-DYNA forum, Bamberg (2005)

13. Directive 2005/66/Ec Of The European Parliament And Of The Council. Available online at: http://eurlex.europa.eu/LexUriServ/site/en/oj/2005/1_309/ 1_30920051125en00370054.pdf

14. G. Lu, T.X. Yu, Energy absorption of structures and materials, Woodhead Publishing Limited, Cambridge, England, 2003, p. 424

15. I. Das, Nonlinear Multicriteria Optimization and Robust Optimality. PhD. Thesis, Dept. of Computational and Applied Mathematics, Rice University, Houston, U.S.A (1997)

16. LS-DYNA Theory Manual, ed. J.O. Hallquist, Livermore Software Technology Corporation, 2006

17. N. Stander, W. Roux, T. Eggleston, K. Craig, LSOPT user's manual. Livermore Software Technology Corporation (2006), p. 425

18. MATLAB Help System, The MathWorks, Inc. (2004) 